\title{
BASE DE CRANEO: ANATOMIA Y PATOLOGIA TUMORAL. REVISION CONCEPTUAL
}

\author{
Drs. Raúl Valenzuela P, Evelyn Ebensperguer $\boldsymbol{P}$.
}

Departamento de TAC y RM, Hospital FACH

\begin{abstract}
Objective. Skull Base Tumors (SBT) span a spectrum from congenital lesions to malignant neoplasms The purpose of this study was to summarize the most relevant anatomical landmarks and to display and organize in a simple manner the SBT and perineural tumoral spread (PTS). Materials and methods. The study displays a selection of cases selected based on teaching value gathered by the author in the 1997-2002 period. Results. SBT can be classified in three main groups: 1-Intrinsic SBT: includes Chordoma, Chondrosarcoma, Meningioma, Neural Sheath Tumors and Metastasic Lesions. 2SBT spreading from below: Includes Nasopharyngeal Carcinoma, Nasopharyngeal Juvenile Angiofibroma and Paranasal Sinus Tumors. 3-SBT from above: It is refers mainly to Pituitary gland macroadenoma. PTS occurs most commonly associated with adenoid cystic and squamous cell carcinomas originated at the Head and Neck region. Most commonly involves $n V$ and nVII. The most important pathway of PTS through the Skull Base is the maxillary division of the trigeminal nerve (nV2). Conclusion. An adequate knowledge of the anatomy, pathology and spread patterns is an important tool for therapy planning and follow-up care.
\end{abstract}

Key words: Neoplasm, Skull base.

Resumen: Objetivo. Revisar la anatomía de la base de cráneo, sistematizar los tumores (TBC) que la afectan y analizar la diseminación perineural (DPN). Material y método. Revisión del archivo recolectado por los autores entre los años 1997-2002 y de casos publicados Resultados. Los TBC se pueden clasificar en: 1) Lesiones intrínsecas como el cordoma, condrosarcoma, meningioma, tumores de la vaina neural y metástasis. 2) TBC que parten des-

Valenzuela R, Ebensperguer E. Base de cráneo: Anatomía y patología tumoral. Revisión conceptual. Rev Chil Radiol 2002; 8: 170-176.

Correspondencia: Dr. Raúl Valenzuela P.

Avda. Pdte. Riesco 6470, Las Condes, Santiago, Chile. de abajo en los cuales se incluyen el carcinoma nasofaríngeo, el angiofibroma juvenil nasofaríngeo y tumores de los senos paranasales. 3) TBC que la comprometen desde sitios por sobre ella y que se refiere principalmente al macroadenoma hipofisiario. La DPN se observa asociada a tumores adenoideos quisticos y carcinomas de células escamosas originados en la región de cabeza y cuello. Compromete comúnmente $n V$ y $n V I I$. Las vías mas importantes de DPN es la división maxilar del nervio trigémino (nV2). Conclusión. Un adecuado conocimiento de la anatomía, patología y vías de diseminación es una importante herramienta en la planificación de la terapia y seguimiento de los TBC.

Palabras claves: Base de cráneo, Tumores

\section{Introducción}

La Base del cráneo (BDC) separa y conecta territorios quirúrgicos como lo son las estructuras intracraneanas con el oído, las cavidades nasales, los senos paranasales, orbitas y cuello suprahioideo.

La mayoría de cirujanos de cabeza y cuello se sienten cómodos bajo la BDC y los neurocirujanos sobre ella, por lo que es común una aproximación conjunta a la patología que la afecta.

La técnica de estudio incluye tomografía computada (TC) con cortes coronales y axiales de 2-4 $\mathrm{mm}$ sin y con contraste intravenoso, con presentación en ventana ósea y de partes blandas. La TC caracteriza bien los limites de destrucción ósea y mal el compromiso intracraneano. La RM debe incluir secuencias coronales y axiales T2-TSE, T1 de alta resolución y T1 con gadolinio y saturación grasa. En comparación con la TC aporta mayor información respecto a la diseminación perineural, leptomeningea y el compromiso intraxial.

\section{Objetivo}

Esta presentación pretende mostrar una visión sinóptica sistematizada de la patología tumoral de base de cráneo, haciendo referencia a la anatomía y rutas de diseminación. 
Anatomía de la base de cráneo (Figura 1)

A.- Estructuras óseas: La BDC esta formada por los huesos frontal, etmoides, esfenoides, temporal y occipital.

El frontal forma la plataforma orbitaria y el etmoides la lamina cribosa y la crista galli en la región anteromedial.

El esfenoides comunica el cerebro, con las orbitas, cara y cuello suprahioideo. Forma la parte superior del clivus, las alas mayores, las alas menores y los procesos pterigoideos.

El plano esfenoidal es la superficie anterosuperior que articula con la lamina cribosa anteriormente y es continuo lateralmente con las alas menores y posteriormente con las clinoides anteriores.

El sulcus quiasmático es la depresión entre el plano esfenoidal y el tubérculo selar.

El clivus es la parte de la BDC que conecta el dorso selar y foramen mágnum. Esta formado por parte del cuerpo del esfenoides y el basioccipucio. Inferiormente esta limitado por el nasofarinx.

A cada lado de la glándula pituitaria se encuentran los senos cavernosos. En su pared lateral transitan los pares craneanos (n) nIII, nIV, nV1, nV2. La carótida, el simpático y $\mathrm{nVI}$ van en la sustancia del seno.

Foramenes de importancia desde adelante hacia atrás y de medial a lateral son el rotundum, el ovale y el espinoso. El foramen rotundum, en la base del ala mayor, comunica hacía atrás con la fosa de Meckel y hacia adelante con la fosa pterigopalatina, fisura orbitaria inferior y canal infraorbitario. El foramen ovale, en la parte posterior del ala mayor, comunica verticalmente la fosa de Meckel con el espacio masticatorio del cuello suprahioideo. Se encuentra en relación posterolateral al foramen rotundum. El foramen espinoso es el mas lateral y posterior.

Otro foramen importante es el lacerum en la parte medial y posterior del esfenoides.

Hay varios foramenes que se identifican en forma variable. El único foramen identificado en forma constante en el cuerpo del hueso esfenoidal es el canal vidiano, en situación anteromedial al foramen lacerum e inferomedial al foramen rotundum, conecta el foramen lacerum con la fosa pterigopalatina. Contiene al nervio vidiano que es la continuación del petroso superficial mayor, rama parasimpática del facial (nVII) después de su unión con el petroso profundo, rama simpática del plexo carotideo. También pasa a través de él, la arteria vidiana rama de la maxilar interna.

El occipital forma el piso de la fosa craneana posterior. Lo integran: a) El basioccipucio, que incluye el clivus y los tubérculos yugulares. b) El exoccipucio que incluye los cóndilos occipitales. c) El supraoccipucio.
EL foramen magnum es la mayor apertura de la base y pasan por él, la medula oblongata, las meninges, arterias vertebrales y nervios espinales accesorios.

El canal hipogloso, que contiene $n X I I$, se ubica anteriormente al condilo occipital en ambos lados.

El temporal incluye los peñascos y mastoides

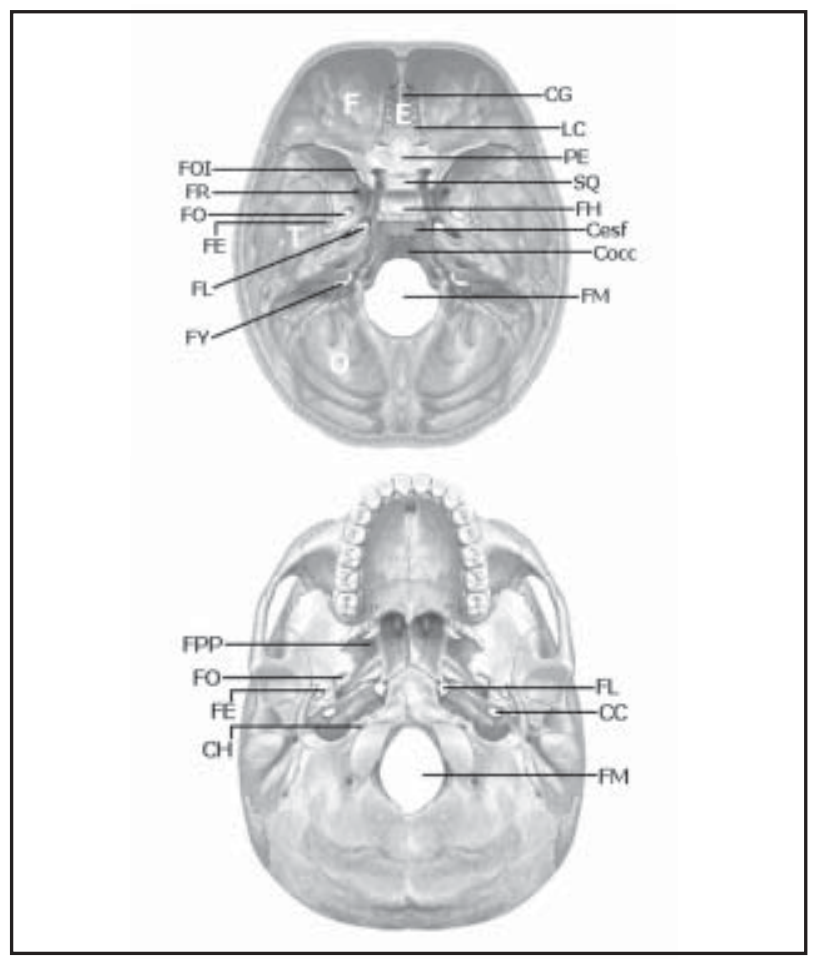

Figura 1 a,b. Anatomía normal base de cráneo vista superior (a) e inferior (b). Se demuestran los huesos frontal (F), etmoidal (E) en el cual se identifica la lamina cribosa ( $L C)$ y la crista galli (CG), temporal (T) y occipital (O). En el esfenoides se identifican el plano esfenoidal (PE), el sulcus quiasmatico (SQ) y la fosa hipofisiaria (FH). En el clivus se muestran su parte esfenoidal (Cesf) y occipital (Cocc). Se identifican además los foramenes de mayor importancia. FR: foramen rotundum, FO: foramen ovale, FE: foramen espinoso, FL: foramen lacerum, FM: foramen magnum. En la vista inferior se demuestran además el canal carotideo (CC), canal hipogloso (CH) y la fosa pterigopalatina (FPP) (modificado de referencia 9)

\section{B.- Aperturas mayores de BDC: Ubicación y con- tenido (Figuras 2 y 3 ) \\ Lamina cribosa ubicada en la parte central de la fosa anterior contiene el nervio olfatorio (nl) \\ Canal óptico en el ala menor de esfenoides con- tiene el nervio óptico (nll).}

Fisura orbitaria superior entre el ala mayor y menor del esfenoides comunica la fosa craneana media con la orbita y especialmente el seno cavernoso y la orbita ${ }^{(1,2)}$. A través de ella pasan nIll, nIV, nV1 y nVI. 
Foramen rotundum ubicada en fosa craneana media contiene $\mathrm{nV} 2$ o maxilar.

Foramen ovale ubicado en la fosa craneana media contiene $\mathrm{nV} 3$ o mandibular.

Foramen lacerum ubicado en la base del proceso pterigoides medial, contiene la rama meníngea $\mathrm{nV} 3$.

Canal Vidiano o pterigoideo en el hueso esfenoidal en situación inferomedial al foramen rotundum. Contiene el nervio Vidiano y la arteria vidiana.

Canal carotideo ubicado en el peñasco. Contiene la carótida interna y el simpático.

Foramen yugular ubicado entre el peñasco y el hueso occipital, esta formado por una: Pars nervosa que contiene el nervio glosofaringeo (IX), nervio de Jacobsen rama IX y una Pars Vascularis: Vago X, espinal XI y nervio de Arnold (rama X).
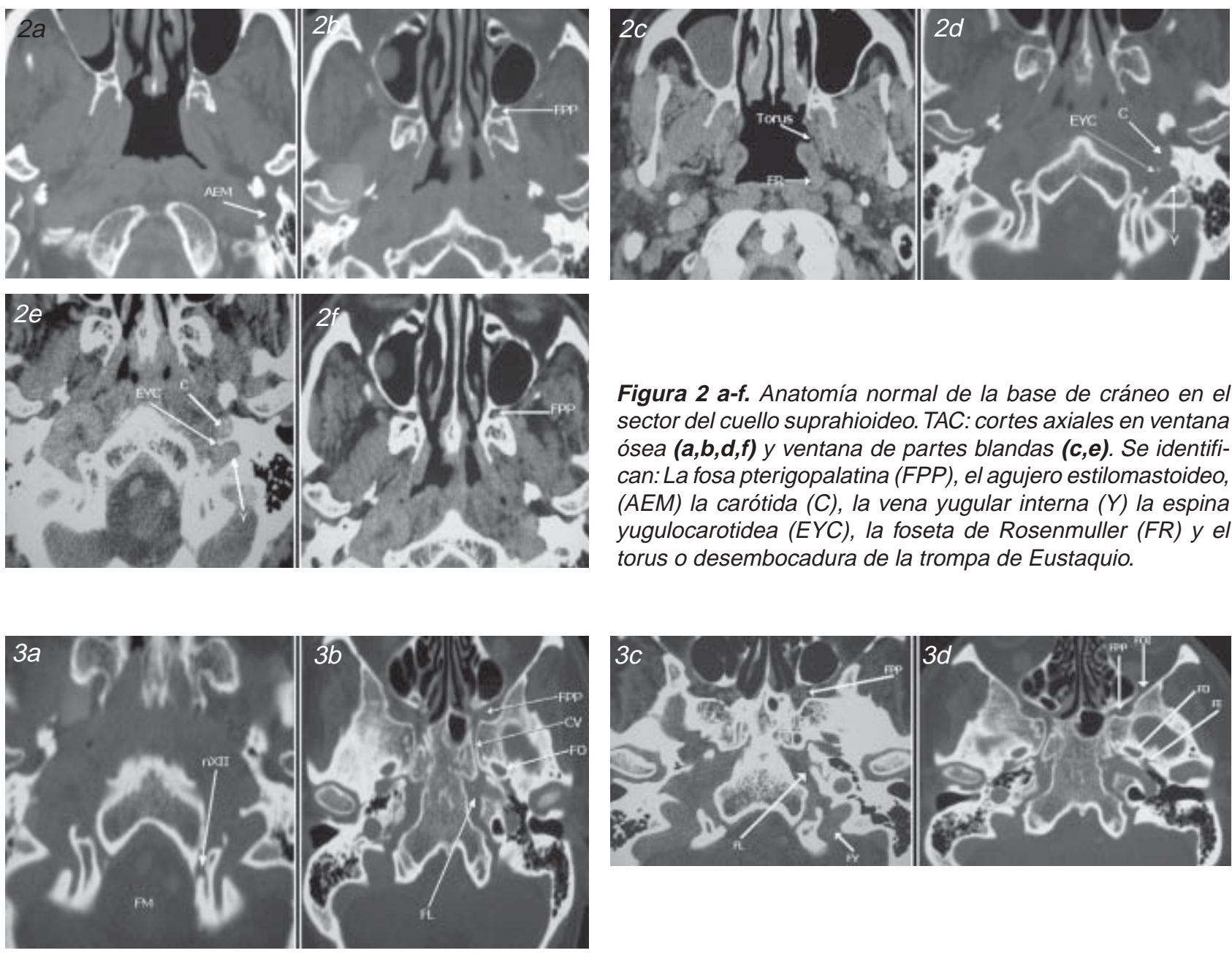

Figura 2 a-f. Anatomía normal de la base de cráneo en el sector del cuello suprahioideo. TAC: cortes axiales en ventana ósea $(\boldsymbol{a}, \boldsymbol{b}, \boldsymbol{d}, \boldsymbol{f})$ y ventana de partes blandas $(\boldsymbol{c}, \boldsymbol{e})$. Se identifican: La fosa pterigopalatina (FPP), el agujero estilomastoideo, $(A E M)$ la carótida $(C)$, la vena yugular interna $(Y)$ la espina yugulocarotidea (EYC), la foseta de Rosenmuller (FR) y el torus o desembocadura de la trompa de Eustaquio.

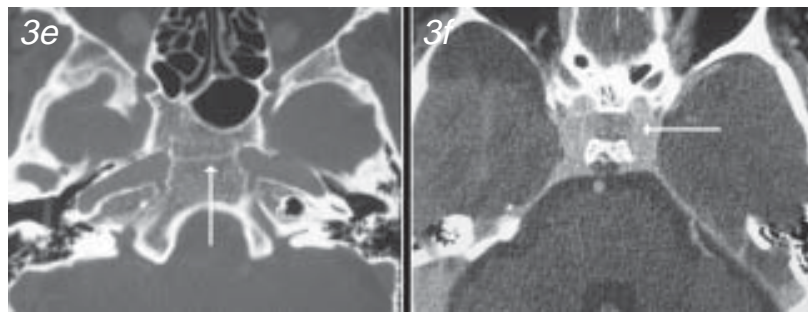

Figura 3 a-f. Base de cráneo anatomía normal. TAC en cortes axiales demostrando: El canal hipogloso ( $\mathrm{X} X \mathrm{II})$, el foramen magnum (FM), la fosa ptegopalatina (FPP), el canal vidiano (CV), los foramenes oval (FO), lacerum ( $F L)$ y yugular ( $F Y)$, la fisura orbitaria inferior (FOI), y el foramen espinoso (FE). Se demuestran además el clivus (flecha vertical en (e)) y el seno cavernoso (flecha horizontal en (f)). 
línea media, sin borde esclerótico, con calcificaciones o restos óseos prominentes. Puede alcanzar al hueso temporal o al troncoencefalo. Presenta áreas que no se refuerzan con contraste y también áreas quisticas. En RM se ven brillantes en T2 lo que permite distinguir su relación con el seno cavernoso ${ }^{(3)}$.

I b. Condrosarcoma: El $6.5 \%$ de estos tumores se originan en la región de cabeza y cuello. Suelen originarse en la fisura o sincondrosis petroccipital. Se extienden desde aquí comprometiendo el clivus y el peñasco.

El aspecto radiológico depende de la cantidad de tejido condroideo. Las calcificaciones son frecuentes y características. La señal es alta en T2 y presentan leve reforzamiento con Gd. Las calcificaciones se identifican en TAC mejor que en RM.

El condrosarcoma tiene mejor pronostico que el cordoma. El condrosarcoma suele ser lateral y el cordoma central.

Existe una variedad mixta o intermedia que es el Cordoma-condroide o Condrocordoma, que suelen originarse de línea media (Figura 4)(4).

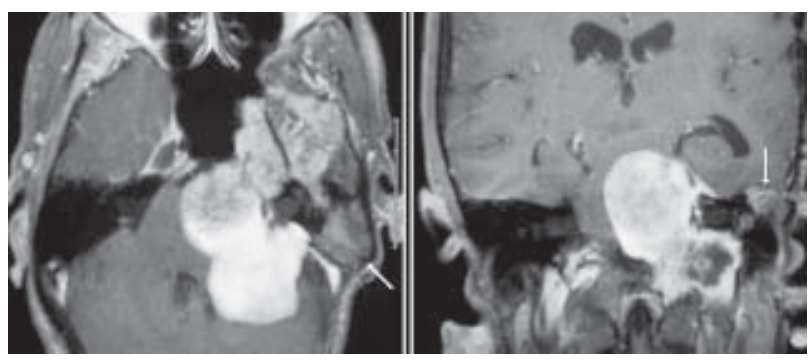

Figura 4 a,b. Cordoma Condroide o Condrocordoma. RM T1 con Gd en cortes axial (a) y coronal (b). Se observa compromiso de fosa posterior y media manifestado como masa de señal hiperintensa. Hay compromiso del espacio prevertebral, fosa de Rosenmuller, ángulo pontocerebeloso, hueso temporal, ganglio de Gasser, seno cavernoso, foramen oval y ápex petroso. La , masa rodea la carótida petrosa y cavernosa, y foramen yugular.Se produce además una mastoiditis obstructiva (flechas).

I c. Meningiomas: Tumores típicamente benignos que se manifiestan entre los 20-60 años. La mayoría se concentra en la convexidad y región parasagital. El compromiso de BDC es el menos frecuente. Cuando comprometen el ala mayor del esfenoides se dividen en 1)-centrales que producen compromiso esfeno-cavernoso y son responsables de múltiples neuropatías craneales y 2)-laterales que son más silentes.

Pueden originarse o seguir a los nervios a través de los forámenes.

En TAC pueden observarse calcificaciones y se contrastan en forma importante. En RM son isointensos al cerebro y captan Gd significativamente demostrando una «cola dural» que aun cuando ca- racterística suele observarse en otros tumores. La evaluación especifica de los meningiomas esfenocavernosos requiere RM y TAC (Figura 5)(5,6).

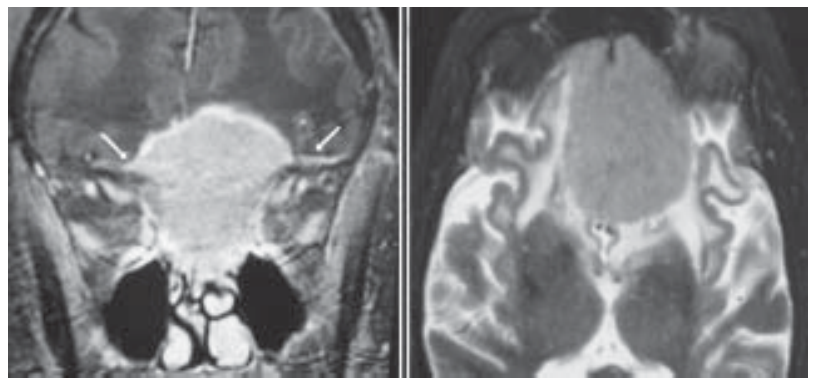

Figura 5 a,b. Meningioma olfativo RM T2 cortes coronal (a) y axial (b) Este tumor simula un estesioneuroblastoma. Se observa una "cola dural" (flechas en a).

\section{I d.- Tumores neurales: Schwannomas} (neurilemomas) se originan en vainas neuronales, pero él más frecuente en la base de cráneo tiene relación con el nervio trigémino. Son raros, los que se originan en $n X I I$, III, IV y VI. Los del IX, X y XI se ubican en los limites del foramen yugular.

El Schwannoma trigeminal se puede originar en la fosa de Meckel extendiéndose comúnmente por el foramen oval o rotundum y presentando un aspecto de "reloj de arena". Es frecuente que protruya hacia el seno cavernoso. Los schwannomas pueden presentar componente quistico y bordes precisos. Son hiperintensos en T2, pero no tanto como el líquido cefalorraquideo (Figura 6).

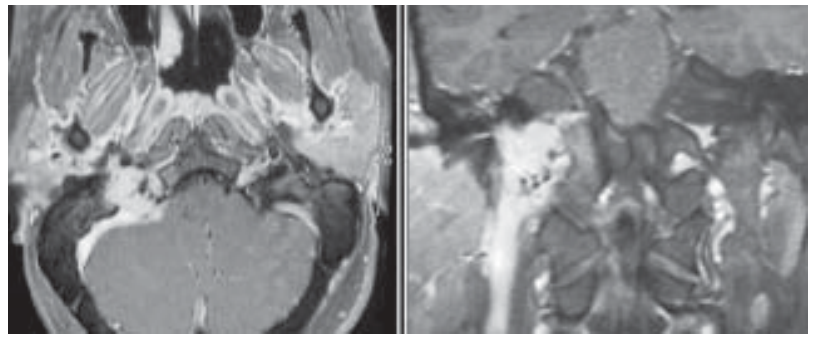

Figura 6 a,b. Tumor del glomus yugular o quemodectomafeocromocitoma extra-adrenal. RM T1 Gd cortes axial (a) y coronal (b). Se observa compromiso yugular y del ángulo pontocerebeloso. Tumor muy vascularizado.

El neurofibroma plexiforme se observa en neurofibromatosis tipo II (NF-II) y es mas invasivo que el Schwannoma. Puede invadir la fisura orbitaria y se puede asociar a hipoplasia esfenoidal que forma parte del compromiso asociado a la NF-II(5,6).

I e.- Metástasis hematógenas: Pueden originarse en tumores de pulmón, riñón, mama, próstata y otros (Figura 7) $)^{(5,6)}$.

I f.- Displasia fibrosa: Es una alteración del desarrollo del hueso en la cual este usualmente esta expandido con conservación del contorno original y con alteración del aspecto medular. El clásico aspecto en 
TAC es de «vidrio esmerilado» (Figura 8). En RM es hipo intenso en T1 y T2 y capta Gadolinio ${ }^{(5,6)}$.
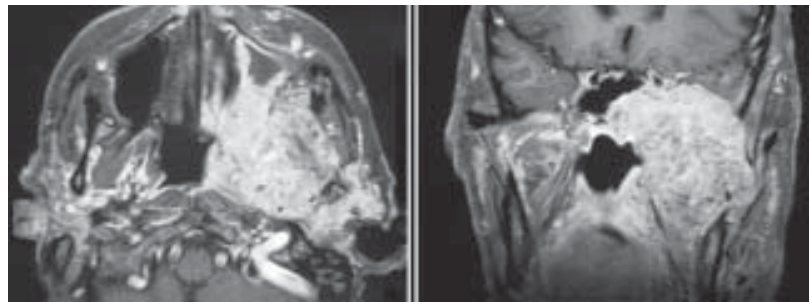

Figura 7 a,b. Carcinoma renal metastático. RM T1 Gd cortes axial (a) y coronal (b). Extensa masa del espacio masticatorio a izquierda con compromiso de la fosa craneana media.

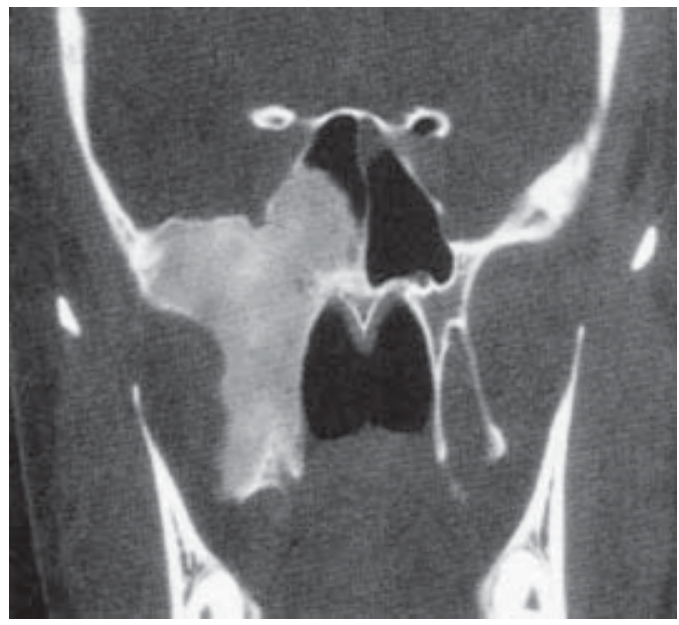

Figura 8. Displasia ósea fibrosa. TAC en corte coronal demostrando afectación del ala mayor del esfenoides, apófisis pterigoidea derecha y ocupación del seno esfenoidal. Se observa un patrón de vidrio esmerilado.(de referencia 5)

I g.- Enfermedad de Paget: De origen incierto y con actividad osteoclastica aumentada. Se produce un hueso más grueso pero más blando. Los hallazgos en BDC tienden a ser líticos especialmente en cápsula otica. Induce platisbasia (Figura 9)(5,6).

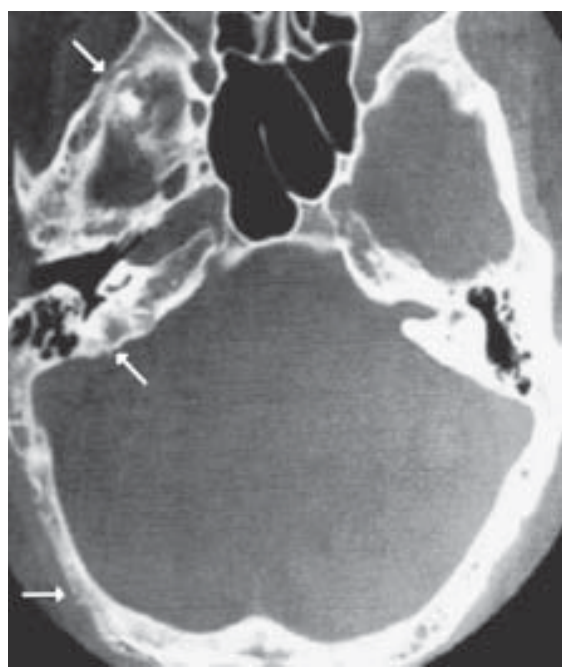

Figura 9. Enfermedad de Paget fase lítica (flechas) comprometiendo, la escama del temporal, el hueso petroso y escama occipital a derecha. (de referencia 5)

\section{II.- Lesiones extrínsecas BDC desde abajo}

II a.-.Carcinoma nasofaringeo: Típicamente invade la base de cráneo central. La resistente fascia faringobasilar limita el crecimiento lateral de este tumor. La fisura petroccipital se encuentra inmediatamente por encima de la fosa de Rosenmuller, donde se origina la mayoría de los carcinomas nasofaringeos; desde aquí se extienden al seno cavernoso y hacia el canal carotideo.

Cuando transgrede la fascia el crecimiento lateral compromete el espacio masticatorio. También implica compromiso de la trompa de Eustaquio y serositis mastoidal obstructiva secundaria. Puede extenderse hacia el canal vidiano y fosa pterigopalatina.

El foramen rotundum usualmente se afecta por compromiso directo y no por diseminación perineural| $^{(5,6)}$ (Figura 10).

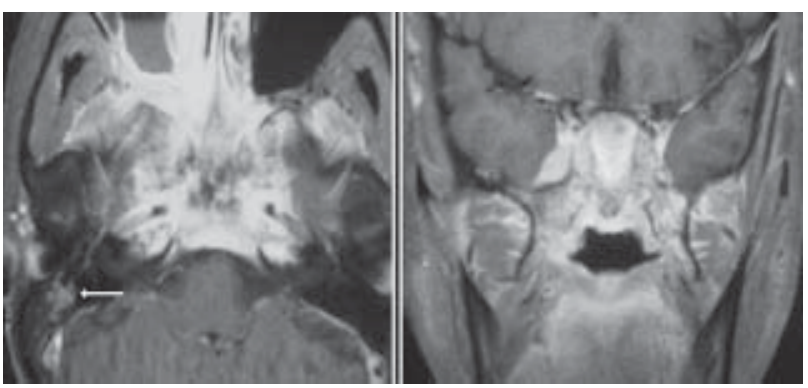

Figura 10 a,b. Carcinoma Rinofaringeo. RM T1 Gd cortes axiales demostrando invasión de: el espacio retrofaringeo, clivus, esfenoides, fosa pterigopalatina derecha, seno cavernoso, ambos canales vidianos. Se observa además mastoiditis obstructiva derecha (flecha en figura a).

II b.- Angiofibroma juvenil nasofaringeo: Es un tumor benigno originado en la cavidad nasal posterior adyacente al agujero esfenopalatino. Ocurre mayoritariamente en jóvenes adolescentes. Se manifiestan por obstrucción nasal y epistaxis. Es un tumor muy vascular, que se refuerza significativamente en TAC y presenta «vacíos de señal» en RM. Casi siempre compromete la fosa perigopalatina la que se ensancha y pierde su contenido graso. Remodela los procesos pterigoideos y los senos maxilares en su parte posterior. Invade el seno cavernoso pudiendo permanecer epidural.

Desde la fosa pterigopalatina alcanza la fosa infratemporal o se extiende hacia la orbita por medio de la fisura orbitaria inferior. Se nutre usualmente por la arteria faringea ascendente, rama terminal de la arteria maxilar interna, a su vez rama de la carótida externa $^{(5,6)}$ (Figura 11).

\section{III.- Lesiones extrínsecas de BDC desde arriba} III a.- Tumores malignos: Pueden afectar la BDC tumores cerebrales, glioma hipotalamicoopticoquiasmático. 

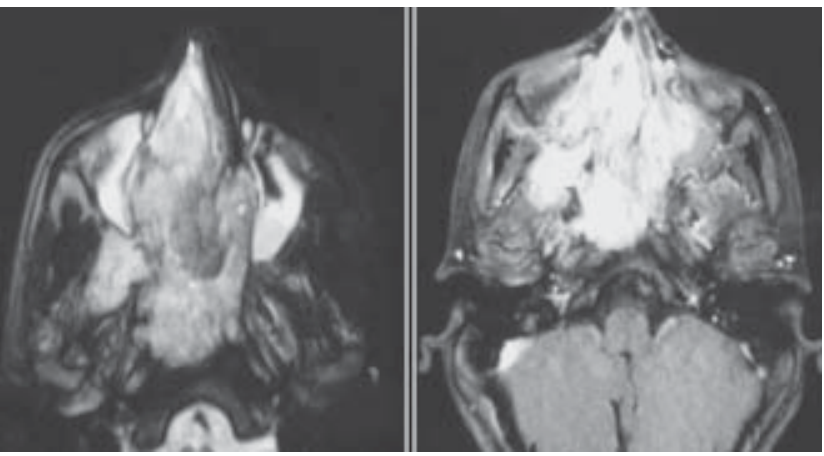

Figura 11 a,b. Angiofibroma Juvenil Nasofaringeo. RM TSE (a) y $T 1 \mathrm{Gd}$ (b) en cortes axiales, se demuestra extenso compromiso de cavidad nasal y ocupación de la fosa pterigopalatina.

III b.- Tumores benignos: Meningioma, tumor de la vaina neural, quiste dermoide, adenoma pituitario.

Adenoma pituitario: De las variedades existentes merecen mención la variedad invasiva y la variedad infraselar. La mayoría de los adenomas crece cefalicamente y cuando transgrede el piso selar y/o invade los senos cavernosos es considerado invasivo.

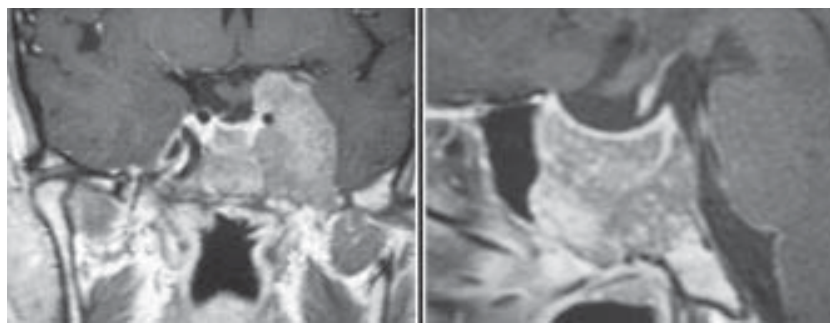

Figura 12 a,b. Adenoma invasivo hipofisiario RM T1 con Gd, cortes coronal (a) y sagital (b), se demuestra un franco compromiso infra y paraselar con afectación del seno cavernoso a izquierda.
Pueden crecer considerablemente e invadir la nasofaringe y la cavidad nasal. A diferencia de un carcinoma invasivo, cuando el seno cavernoso es invadido, la carótida es rodeada pero no estenosada en el caso del adenoma y sí en carcinoma.

La variedad infraselar es extremadamente rara e implica indemnidad del piso selar con tumor en el hueso esfenoidal(7) (Figura 12).

III c.- Malformaciones Congénitas: Céfalocelemeningocele-encefalocele, quiste aracnoidal.

Cefalocele: Es una protrusión de contenido intracraneal por un defecto en el cráneo. Si contiene meninge y LCR es llamado meningocele, si además se agrega cerebro es un encefalocele. La mayoría compromete la calota y solo el $20 \%$ compromete BDC anterior o central (céfaloceles basales).

Los basales se clasifican en: 1-transetmoidal, 2esfenoetmoidal, 3-esfenorbital, 4-transesfenoidal y 5 -esfenomaxilar. Los tipos 1,2 y 4 son centrales y los 3 y 5 son laterobasales ${ }^{(5,6)}$.

III d.- Anomalías Vasculares: Aneurisma carotidocavernoso, malformación arteriovenosa dural.

\section{Diseminación perineural (Tabla I)}

Se observa mas frecuentemente en carcinomas adenoideos quisticos y escamosos. También puede ocurrir en otras neoplasias malignas tales como las salivales, linfoma, melanoma, carcinoma basocelular y rabdomiosarcoma. También es visible en neoplasias benignas o malignos de vainas neurales, meningiomas y angiofibromas juveniles.

La diseminación perineural de los tumores de cabeza y cuello ocurren con relación a nervios $\mathrm{V}$ y VII La ruta de diseminación perineural más impor-

Tabla I. Diseminación tumoral perineural.

Area Comprometida

Apex orbitario

Fosa orbitaria superior

Seno cavernoso

Fosa pterigopalatina

Foramen rotundum

Seno cavernoso

Foramen oval

Seno cavernoso

VII nervio intratemporal

Canal Vidiano

Fosa pterigopalatina

Nervio petroso superior
Nervio

V1

(oftálmico)

V2

(maxilar)

V3

(mandibular)

VII

Vidiano

\section{Origen tumor}

Orbita

Seno etmoidal

Seno frontal

Maxilar, Paladar

Nasofarinx

Cara media

Espacio masticador

Parafaringeo, Mandíbula

Parótida

Hueso temporal

Canal auditivo externo

Nasofarinx

Glándulas lacrimales

Áreas inervadas por V2 y VII

mayor y profundo 
tante desde y a través de la BDC central es nV2. Inerva con sus ramas infraorbitaria, palatina y alveolar superior la cara, paladar duro y seno maxilar. Típicamente la diseminación es retrograda hacia el cráneo, hacia la fosa de Meckel y desde ahí anterograda hacía el espacio masticatorio(5,6,8) (Figura 13).

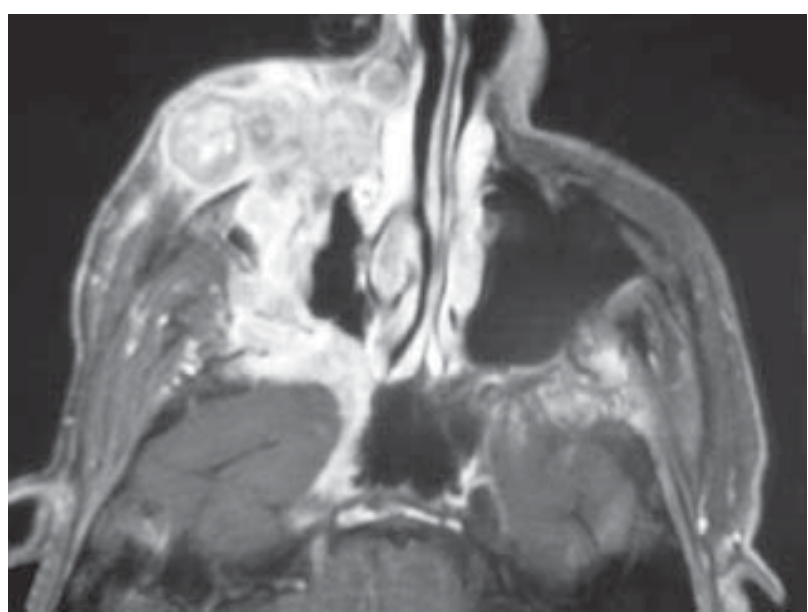

Figura 13. Melanoma periorbitario derecho. RM T1 Gd con saturación grasa, corte axial que demuestra diseminación perineural posterior a través de la fisura infraorbitaria (nV2).

\section{Conclusión}

El cabal conocimiento de la anatomía, patología y patrón de diseminación de la patología tumoral de
BDC, es una herramienta central en la planificación de la terapia tumoral y su seguimiento.

\section{Bibliografía:}

1. Lustrin E.S, Robertson R.L., Tilak S. Normal anatomy of the skull base. Neuroimaging Clinics of North America. 1994; 4: 465-478.

2. Harnsberger H.R., Dillon W.P. Suprahyoid neck ad central skull base: Normal anatomy and pathologic processes. Syllabus: A special course in head and neck radiology. RSNA Publications 1996.

3. Weber AL., Liebsch NJ., Sanchez R, Sweriduk ST, Jr. Chordomas of the skull base: Radiologic and clinical evaluation. Neuroimaging Clinics of North America. 1994; 4: 515-528.

4. Brown E, Hug EB, Weber AL. Chondrosarcoma of th skull base. Neuroimaging Clinics of North America 1994; 4: 529-542.

5. Osborn AG. Diagnostic Neuroradiology.484-511. Mosby Year Book, Inc. MA USA 1994.

6. Peter M.Som, Hugh D. Curtin. Head and Neck Imaging 1233-1299. Mosby Year Book, Inc. MA USA 1994

7. Felsberg GJ, Tien RD. Sellar and parasellar lesions involving the skull base. Neuroimaging Clinics of North America 1994; 4: 543-560.

8. Caldemeyer KS, Mathews VP, Righi PD, Smith R.R. Imaging Features and Clinical Significance of Perineural Spread or Extension of Head and Neck Tumors. Radiographics 1998; 19: 97-110.

9. Netter FH. Atlas de anatomía humana. Masson S.A. 1996 New Yersey. USA. 